\title{
Soft mode parameter as an indicator for the activation energy spectra in metallic glass
}

\author{
Shan Zhang a , Chaoyi Liu ${ }^{\mathrm{b}}$, Yong Yang ${ }^{\mathrm{c}}$, Yue Fan ${ }^{\mathrm{b}}$, Pengfei Guan ${ }^{\mathrm{a}, *}$ \\ a Beijing Computational Science Research Center, Beijing, China \\ ${ }^{\mathrm{b}}$ Department of Mechanical Engineering, University of Michigan, Ann Arbor, USA \\ ${ }^{\mathrm{c}}$ Centre for Advanced Structural Materials, Department of Mechanical and Biomedical Engineering, City \\ University of Hong Kong, Hong Kong, China
}

Corresponding author: Pengfei Guan*

Email:pguan@csrc.ac.cn 
ABSTRACT: The activation energy $\left(E_{\mathrm{A}}\right)$ spectra of potential energy landscape (PEL) provides a convenient perspective for interpreting complex phenomena in amorphous materials; however, the link between the $E_{A}$ spectra and other physical properties in metallic glasses is still mysterious. By systematically probing the $E_{\mathrm{A}}$ spectra for numerous metallic glass samples with distinct local geometric ordering, which correspond to broad processing histories, it is found that the shear modulus of the samples are strongly correlated with the arithmetic mean of the $E_{\mathrm{A}}$ spectra rather than with the local geometrical ordering. Furthermore, we studied the correlation of the obtained $E_{\mathrm{A}}$ spectra and various wellestablished physical parameters. The outcome of our research clearly demonstrates that the soft mode parameter $\Psi$ and the $E_{\mathrm{A}}$ spectrum are correlated; therefore, it could be a good indicator of metallic glass properties and sheds important light on the structure-property relationship in metallic glass through the medium of PEL.

TOC Graphic

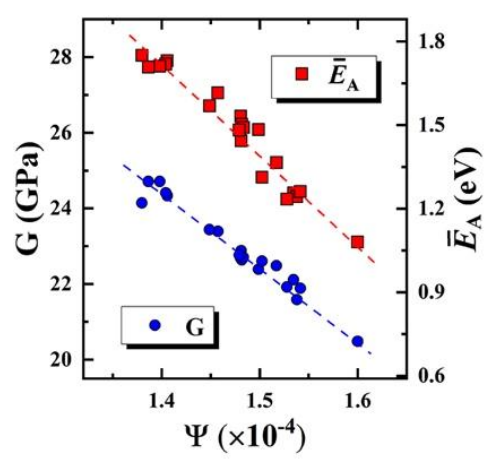


Metallic glasses (MGs), as metastable systems, can provide ample opportunity for property control that can be used in structural and functional applications ${ }^{1-8}$. However, due to their inherent nature of disordered atomic packing and, consequently, the absence of conventional defects with distinctive topologies (e.g. dislocations, grain boundaries, etc.), it is very difficult to establish a "crystal clear" structure-property relationship for MGs. In fact, the definition of "defects" in disordered materials are obscure and not unique: examples include free volume (FV) ${ }^{9-10}$, shear transformation zones (STZ) ${ }^{11-15}$, Voronoi tessellation ${ }^{16-17}$, flow units ${ }^{18-20}$, soft modes/spots ${ }^{21-23}$, regions with large fluctuations of atomic level stresses ${ }^{24-26}$, etc. Tremendous efforts have been made in the past to examine the correlations between the local atomic relaxations and those "defects" $21,27-30$. On the other hand, it is known that many macroscopic properties of MGs (e.g. heat release/absorption ${ }^{31-32}$, homogeneous deformation ${ }^{18}$, etc.) are actually governed by the overall structural distributions and activation energy $\left(E_{\mathrm{A}}\right)$ spectra, rather than by some extreme "defects", which are located near the ends of the spectra. From the PEL perspective, the $\alpha$ relaxation process can be pictured as the transitions between the deep 'metabasins', whereas the $\beta$ relaxation process refers to the elementary hopping event between the 'sub-basins'coded within a metabasin. These processes are related to many important properties, such as glass transition, deformation, aging and rejuvenation etc., of MGs. Systematically analyzing the activation energy $\left(E_{\mathrm{A}}\right)$ spectra for these processes are extremely helpful for understanding some critical phenomena ${ }^{33}$, and establishing the microscopic structure-property connections. Therefore, important questions that follow and yet remain open are: how does the $E_{\mathrm{A}}$ spectra correlate with the physical properties of interest in MGs, and what are the most effective structural indicators in determining MGs' $E_{\mathrm{A}}$ spectra?

In this work, molecular dynamic (MD) simulations that combine potential energy landscape 
(PEL)-sampling technique were employed to investigate the physical properties and systematically probe the $E_{\mathrm{A}}$ spectra for numerous MGs samples with distinct local geometry or short-range order (SRO) behaviors. These samples are prepared over broad processing histories in the cooling ratepressure space. It is found that the shear modulus $G$ is strongly correlated with the arithmetic mean of $E_{\mathrm{A}}$ spectra rather than with the local geometry ordering (such as the fraction of icosahedron clusters), even though the processing history does induce pronounced local structure changes. Focuses are placed on how those $E_{\mathrm{A}}$ spectra are correlated with the five widely used representative parameters, including the overall density, the inherent structure energy $\left(E_{\mathrm{IS}}\right)$, the two-body entropy $\left(\mathrm{S}_{2}\right)$, the soft-mode parameter $(\Psi)$, and the local configurational anisotropy $(|\vec{u}|)$. It is found that a sample's $E_{\mathrm{IS}}$ and soft-mode parameter $(\Psi)$ exhibit the best correlation with the arithmetic mean $\bar{E}_{\mathrm{A}}$. By further quantifying the differences between each pair of obtained $E_{\mathrm{A}}$ spectra and mapping them into various measure spaces, we demonstrate that, among those five representative parameters, the soft-mode parameter performs the best in describing a sample's $E_{\mathrm{A}}$ spectrum and, thereby, the shear modulus G.

A $\mathrm{Cu}_{50} \mathrm{Zr}_{50}$ model system with the realistic embedded atom method (EAM) potential ${ }^{17,34}$ is considered in the present work as a prototypical MG. The details of our model systems and activation-relaxation technique (ART) method are presented in the Simulation Methods and Supporting Information. In brief, the 4000 -atom glass samples are prepared by systematically tuning the hydrostatic stress $(0,5,10,15,20 \mathrm{GPa})$ and the cooling rate $\left(10^{10}, 10^{11}, 10^{12}, 10^{13} \mathrm{~K} / \mathrm{s}\right)$. To be more specific, the preparation process for each sample consists of two steps (Fig 1a): (i) quench the initial supercooled liquid at the prescribed pressure $\left(P_{i}\right)$ and cooling rate condition to $1 \mathrm{~K}$; (ii) release the applied pressure and let the system relax at $1 \mathrm{~K}$ for $10 \mathrm{~ns}$. The state of metallic glasses is tuned by the cooling rate and prescribed pressure $P_{i}$ during the quenching process, even 
the density changes are slight $(\sim 1 \%)$. Both the cooling rate and the $P_{i}$ introduce changes to the total radial distribution functions (Fig. S1), but the influence of $P_{i}$ is more pronounced than cooling rate. This indicates that the atomic structure, especially the local atomic packing behavior of these samples should be different. As shown in Fig. 1b, the fractions of $<0,0,12,0>$ cluster (denoted as $f$ $<0,0,12,0>$ ) of samples increases nearly three times due to the high-pressure effect, which is comparable to the results in ref. ${ }^{35}$. The $f_{\text {ico }}$ is tuned in a wide range $(0.02 \sim 0.12)$ by varying $P_{i}$ and the cooling rate. This behavior suggests that these MG samples have distinct local geometries or short-range orders (SROs). Therefore, these samples form a system with similar densities but vastly different atomic configurations. Their physical properties, such as the shear modulus $\mathrm{G}$ and the activation energy $\left(E_{\mathrm{A}}\right)$ spectra etc. will be investigated to reveal the correlations, if any, between the structural features and the physical properties of interest.

Since the shear modulus is at the heart of may key properties of MGs, we focus on the correlation between $\mathrm{G}$ and other parameters. Figure 1c shows the correlation between the $f<0,0,12,0>$ and the calculated shear modulus $G$ of the investigated samples. It is clear that we do not obtain the collapse of data for these samples. This means that the local geometrical order is not appropriate for describing the mechanical properties of MGs, as mentioned in the previous studies ${ }^{35-36}$. According to the PEL perspective, we may obtain the collapse of data $G$ as a function of the intrinsic behaviors of the $E_{\mathrm{A}}$ spectra, such as the arithmetic mean of the $E_{\mathrm{A}}$ spectra, $\bar{E}_{\mathrm{A}}$. By performing the ART in our MD simulations, the $E_{\mathrm{A}}$ spectra of 20 samples in Fig. $1 \mathrm{~b}$ can be monitored. The related arithmetic mean $\bar{E}_{\mathrm{A}}$ is calculated by $\bar{E}_{\mathrm{A}}=\left\langle\sum_{i=1}^{N_{\text {event }}} E_{\mathrm{A}, \mathrm{i}}\right\rangle$, where $\mathrm{N}_{\text {event }}$ is the number of valid events in the ART methord(see the Supporting Information for more details ). As we plotted $\mathrm{G}$ as a function of $\bar{E}_{\mathrm{A}}$ (Fig. 1d), it is amazing to find the collapse of data for all samples. In theory, this behavior provides the direct evidence for the intrinsic correlation between the PEL 
and mechanical properties. In concept, this finding also implies that the systematical study of PEL may build a bridge between the structure and properties of MGs on a quantitative basis. Now, the question is: Is there a structural parameter that is correlated with the $E_{\mathrm{A}}$ spectra.

To address the above question, we analyzed the $E_{\mathrm{A}}$ spectra of each sample. Fig. 2a shows the $E_{\mathrm{A}}$ spectra of 20 samples considered in the present study, and the corresponding cooling rates and hydrostatic stresses are also marked in the plot. It can be seen that, as the cooling rate increases (i.e. comparing the panels in the same row), the $E_{\mathrm{A}}$ distribution gradually shifts towards the left side and develops a fat tail in the low $E_{\mathrm{A}}$ region. This is not surprising, because a higher cooling rate means the sample has less time to relax and therefore is quenched in a less stable state that is more susceptible to perturbations ${ }^{32,37}$. On the contrary, the pressure effects (i.e. comparing the panels in the same column) are much more complex and seem to couple with the cooling rates. For example, under the cooling rate of $10^{10} \mathrm{~K} / \mathrm{s}$, the $E_{\mathrm{A}}$ spectra are insensitive to the pressure; while under the cooling rate of $10^{13} \mathrm{~K} / \mathrm{s}$, the $E_{\mathrm{A}}$ spectra clearly shift towards the low- $E_{\mathrm{A}}$ end as the pressure increases. This is somewhat surprising because, intuitively, a higher pressure would squeeze out free volumes, thus making the sample more stable. It is worth noting that such abnormal dependence on pressure is consistent with the recent studies ${ }^{38}$, which is an interesting topic for future investigation.

To unravel the origin of the complex $E_{\mathrm{A}}$ spectra, we construct the radar plots, as seen in the inset of each panel, which show the relative importance of 5 widely used structural indicators, including the density $(\rho)$, the inherent structure energy $\left(E_{\mathrm{IS}}\right)$, the two-body entropy $\left(s_{2}\right)$, the softmode parameter $(\Psi)$, and the local configurational anisotropy $(|\vec{u}|)$. We would like to note that, while these structural indicators can be defined locally at atomic level (see the Supporting Information), here we focus on their average values in the radar plots and also in the following 
discussions in line with the global nature of the $E_{\mathrm{A}}$ spectrum.

We first examine how the above-mentioned 5 indicators of a sample correlate with the simple arithmetic mean $\bar{E}_{\mathrm{A}}$. To better compare the different measures, a polar coordinate system is employed, and all the 5 panels are presented in the same plot in Fig. 2b. The radial axis represents the magnitude of the arithmetic mean activation energy, $\bar{E}_{\mathrm{A}}$; while the $\left[0^{\circ}, 180^{\circ}\right]$ angular regime is equally divided into five $36^{\circ}$-wide sub-regimes to represent different structural indicators. To be more specific, the $\left[144^{\circ}, 180^{\circ}\right]$ regime represent $\rho$, which is usually used to estimate the free volume of MGs in experiments ${ }^{39}$. As discussed above, it is intuitive that a higher density should correspond to less free volume and, consequently, a larger effective $\bar{E}_{\mathrm{A}}$. However, no such correlation can be observed in the present study and the data points are widely scattered. In the $\left[108^{\circ}, 144^{\circ}\right]$ regime we consider $s_{2}$, which was shown in some prior studies as an effective indicator for slow kinetics ${ }^{40-41}$. Although the $\bar{E}_{\mathrm{A}}$ seems to become larger as $s_{2}$ decreases, which is consistent with the previous studies ${ }^{40}$, yet the overall correlation is rather weak. In the $\left[72^{\circ}, 108^{\circ}\right]$ regime, we consider $|\vec{u}|$, which has drawn much attention in recent studies ${ }^{42-44}$ due to its predictive power for particle rearrangements. As seen by the blue hexagons in Fig. $\mathbf{2 b}$, indeed there is a tendency that a larger $|\vec{u}|$ would increase the likelihood of atom rearrangements (i.e. lower $\bar{E}_{\mathrm{A} \text {.) }}$ But still, the data show a significant scatteredness, suggesting that the correlation is not strong. In the $\left[36^{\circ}, 72^{\circ}\right]$ regime we examine $E_{\mathrm{IS}}$, which has also been widely used as an indicator of glasses' stability ${ }^{32,37,}$ ${ }^{45-46}$. It can be seen that the data points are reasonably well aligned with a diagonal curve, indicating a linear dependence between the two variables in a regular Cartesian coordinate system. Finally, in the $\left[0^{\circ}, 36^{\circ}\right]$ regime we examine $\Psi$, another well-accepted structural measure that has shown strong correlations with atoms' non-affine displacements in amorphous solids ${ }^{28,47}$. As seen by the yellow triangles in Fig. 2b, there is a good linear correlation between $\Psi$ and $\bar{E}_{\mathrm{A}}$. To briefly 
summarize, among the 5 structural indicators considered in the present study, the $E_{\mathrm{IS}}$ and $\Psi$ are correlated very well with the system's $\bar{E}_{\mathrm{A}}$; while the other three measures, including $\rho, s_{2}$, and $|\vec{u}|$, only show weak correlations with $\bar{E}_{\mathrm{A}}$.

It is worth noting that, the hereby considered $\bar{E}_{\mathrm{A}}$ merely represents the simple arithmetic mean calculation of the $E_{\mathrm{A}}$ distribution, However, in real physical and thermally activated processes, the Boltzmann weight factor, $\exp \left[-E_{\mathrm{A}} / k_{\mathrm{B}} T\right]$, has to be considered ${ }^{18,32}$, which renders the calculation of $\left\langle E_{\mathrm{A}}\right\rangle T$-dependent. Therefore, it is more physically meaningful to obtain the probability distribution function $P\left(E_{\mathrm{A}}\right)$, rather than the simple arithmetic mean value, $\bar{E}_{\mathrm{A}}$. The reason is that, if two samples have the same $P\left(E_{\mathrm{A}}\right)$ then they will always have the same performance; however, if they have the same $\bar{E}_{\mathrm{A}}$ then they do not necessarily possess the same $P\left(E_{\mathrm{A}}\right)$, which yield different behaviors under various conditions. To this end, in what follows we will investigate which structural indicator could potentially best describe $P\left(E_{\mathrm{A}}\right)$ with the least uncertainty. As illustrated in Fig. 3a, to quantify the $E_{\mathrm{A}}$ spectra difference between two samples here we define a $\delta$ function as $\delta_{i, j}=\int\left[P_{i}\left(E_{A}\right)-P_{j}\left(E_{A}\right)\right]^{2} d E_{A}$, where $P_{i}\left(E_{\mathrm{A}}\right)$ and $P_{j}\left(E_{\mathrm{A}}\right)$ represent the spectra of samples \# $i$ and $\# j$, selected from the previously obtained dataset in Fig. 2a. In Fig. 3b-f we map the calculated $\delta_{i, j}$ into the 5 different structural spaces. By systemetrical analyzing the contour maps (see the Supporting Information), the distributions of $\delta_{i, j}$ in Fig. 3e and Fig. 3f suggest that $E_{\mathrm{IS}}$ and $\Psi$ are still the two best candidates correlating with $P\left(E_{\mathrm{A}}\right)$. We hypothesize that $E_{\mathrm{IS}}$ might be a more viable option to express $P\left(E_{\mathrm{A}}\right)$ for MG samples with similar density due to the relatively low computational costs. It is worth noting that, some recent attempts to use $E_{\mathrm{IS}}$ as the structural parameter have successfully explained the MGs' exothermic features observed in experiments ${ }^{32}$, ${ }^{48}$, which provide further premises in support of our hypothesis. 
Importantly, through the medium of $E_{\mathrm{A}}$ spectra, we can expect a strong correlation between $\mathrm{G}$ and $E_{\mathrm{IS}}$ or $\Psi$. As shown in Fig. 4a and b, the $\Psi$ presents much better correlation with G, which implies that the soft-mode parameter would probably be a more advantageous indicator for the activation energy spectra in metallic glass. It can be well explained by the intrinsic correlation between the soft-mode parameter and the Debye-Waller factor. On short timescales, the local Debye-Waller factor $\alpha_{i}$ is primarily determined by local structures that can be employed as a structural parameter as well. As proposed by Tong and $\mathrm{Xu}^{47}$ based on the equipartition hypothesis, the soft-mode parameter for individual particles $\Psi_{\mathrm{i}}=\sum_{\mathrm{j}=1}^{\mathrm{dN}-\mathrm{d}} \frac{1}{\omega_{\mathrm{j}}^{2}}\left|\overrightarrow{\mathrm{e}}_{\mathrm{j}, \mathrm{i}}\right|^{2}$ is statistically proportional to the single-particle Debye-Waller factor $\alpha_{i}$ in glass systems. Previous simulations ${ }^{38}$ have shown that the short-time local positional fluctuation $\alpha_{i}$ provides a good predictor of metallic glass properties at low temperature, such as shear modulus G. Thus, the soft-mode parameter $\Psi=$ $\sum_{i=1}^{N} \Psi_{\mathrm{i}}$, where $\mathrm{N}$ is the number of atoms, could be a good predictor of shear modulus $\mathrm{G}$ and the activation energy spectra in metallic glass. Moreover, $\Psi$ is mainly contributed by the atoms with larger $\Psi_{\mathrm{i}}$ which are dominated by the low-frequency modes. It means that the parameter $\Psi$ quantitatively reflects the defective local structures in metallic glass. The strong correlation between $G$ and $\Psi$ implies that the physical properties of metallic glass are sensitive to the geometrically unfavored Voronoi motifs rather than the favored Voronoi motifs, such as icoshedron ${ }^{35-36}$.

In summary, we employ the ART method to directly probe the $E_{\mathrm{A}}$ spectra for a number of $\mathrm{Cu}_{50} \mathrm{Zr}_{50} \mathrm{MG}$ samples that are prepared at different pressure and cooling rate conditions over a broad range of parameter space. It is found that a sample's shear modulus is strongly correlated with its arithmetic mean of $E_{\mathrm{A}}$ spectra rather than the local geometrical ordering. By quantifying the differences between each pair of obtained $E_{\mathrm{A}}$ spectra and mapping them into various widely 
investigated representative parameter spaces, we demonstrate that the soft mode parameter $\Psi$ has the greatest potential to determine a sample's $E_{\mathrm{A}}$ spectrum. Through the medium of PEL, the soft mode parameter $\Psi$ can be proposed as a good predictor of metallic glass properties, such as shear modulus G. Furthermore, the capability of establishing a quantitative description on $P\left(E_{\mathrm{A}}\right)$ is critical, because as discussed above, it determines many macroscopic thermo-mechanical properties of amorphous solids ${ }^{18,31-32}$. Although the present study does not yet give an explicit formulism of $P\left(E_{\mathrm{A}}\right)$, it provides effective guidance towards such a goal. The hereby obtained correlations and uncertainty quantifications, in conjunction with machine learning tools, are expected to enable an analytical and accurate expression of $P\left(E_{\mathrm{A}}\right)$ in the future.

\section{SIMULATION METHODS}

Molecular dynamics simulations have been performed by utilizing the open-source codeLAMMPS $^{49}$. A 4000-atom simulation box, with periodic boundary conditions applied in all three directions, is first equilibrated at a supercooled liquid state with the temperature of $1500 \mathrm{~K}$ and volume of $43 \times 43 \times 43 \AA^{3}$. Then the glass samples are prepared by systematically tuning the hydrostatic stress $(0,5,10,15,20 \mathrm{GPa})$ and the cooling rate $\left(10^{10}, 10^{11}, 10^{12}, 10^{13} \mathrm{~K} / \mathrm{s}\right)$ with the constant number, pressure, and temperature (NPT) ensemble. Then, the activation-relaxation technique (ART) method ${ }^{50-51}$ was employed to probe the PEL of the prepared glassy samples and thereby its $E_{\mathrm{A}}$ spectrum. To initiate the PEL sampling, small random perturbations with a total displacement of $0.5 \AA$ are introduced to a cluster of atoms within the first nearest neighbor distance. Then, the ART searching algorithm will drive the system to leave the current basin (with the curvature criterion of $-0.01 \mathrm{eV} / \AA^{2}$ ) and identify the connecting saddle states (with the force convergence criterion of $0.05 \mathrm{eV} / \AA$ ). By statistically repeating such perturbation and search 
processes with various random seeds and over different positions in the system, the representative $E_{\mathrm{A}}$ spectrum can thus be obtained (see Supporting Information for more details).

\section{Acknowledgments}

This work is supported by (S.Z. and P.G.) the NSF of China (Grants No. 51571011 and U1930402 ) and the MOST 973 Program (No. 2015CB856800). S.Z. and P.G. acknowledge the computational support from the Beijing Computational Science Research Center(CSRC). C.L. and Y.F. acknowledge the support from the U. S. Army Research Office under Grant No. W911NF18-1-0119.

Reference:

1. Padmanabhan, J.; Kinser, E. R.; Stalter, M. A.; Duncan-Lewis, C.; Balestrini, J. L.; Sawyer, A. J.; Schroers, J.; Kyriakides, T. R. Engineering Cellular Response Using Nanopatterned Bulk Metallic Glass. ACS Nano 2014, 8 (5), 4366-4375.

2. Schroers, J. Bulk Metallic Glasses. Phys. Today 2013, 66 (2), 32.

3. Schroers, J.; Kumar, G.; Hodges, T.; Chan, S.; Kyriakides, T. Bulk metallic glasses for biomedical applications. JOM 2009, 61 (9), 21-29.

4. Johnson, W. L. Bulk Metallic Glass, a New Engineering Material Now Commercially Available. Mater. Technol. 1994, 9 (5-6), 94-96.

5. Raskin, D.; Smith, C. H. Chapter 20 - Applications of amorphous metals: progress and prospects A2 - LUBORSKY, F.E. In Amorphous Metallic Alloys, Butterworth-Heinemann: 1983; pp 381-400. 
6. Zinkle, S. J.; Snead, L. L. Designing Radiation Resistance in Materials for Fusion Energy. Annu. Rev. Mater. Res. 2014, 44 (1), 241-267.

7. Mei, X.; Wang, B.; Dong, C.; Gong, F.; Wang, Y.; Wang, Z. Anti-irradiation performance against helium bombardment in bulk metallic glass $\left(\mathrm{Cu}_{47} \mathrm{Zr}_{45} \mathrm{Al} \mathrm{I}_{8}\right)_{98.5} \mathrm{Y}_{1.5}$. Nucl. Instrum. Methods Phys. Res., Sect. $B$ 2013, 307, 11-15.

8. Weber, W. J.; Ewing, R. C.; Angell, C. A.; Arnold, G. W.; Cormack, A. N.; Delaye, J. M.; Griscom, D. L.; Hobbs, L. W.; Navrotsky, A.; Price, D. L.; Stoneham, A. M.; Weinberg, M. C. Radiation Effects in Glasses Used for Immobilization of High-level Waste and Plutonium Disposition. J. Mater. Res. 1997, 12 (08), 1948-1978.

9. Spaepen, F. A microscopic mechanism for steady state inhomogeneous flow in metallic glasses. Acta Metall. 1977, 25 (4), 407-415.

10. Cohen, M. H.; Turnbull, D. Molecular Transport in Liquids and Glasses. J. Chem. Phys. 1959, 31 (5), 1164-1169.

11. Falk, M. L.; Langer, J. S. Dynamics of viscoplastic deformation in amorphous solids. Phys. Rev. E 1998, 57 (6), 7192-7205.

12. Langer, J. S. Dynamics of shear-transformation zones in amorphous plasticity: Formulation in terms of an effective disorder temperature. Phys. Rev. E 2004, 70 (4), 041502.

13. Argon, A. S. Plastic deformation in metallic glasses. Acta Metall. 1979, 27 (1), 47-58.

14. Argon, A. S.; Kuo, H. Y. Plastic flow in a disordered bubble raft (an analog of a metallic glass). Mater. Sci. Eng. 1979, 39 (1), 101-109.

15. Schuh, C. A.; Lund, A. C. Atomistic basis for the plastic yield criterion of metallic glass. Nat. Mater. 2003, 2 (7), 449-452.

16. Sheng, H. W.; Luo, W. K.; Alamgir, F. M.; Bai, J. M.; Ma, E. Atomic packing and short-to-mediumrange order in metallic glasses. Nature 2006, 439 (7075), 419-425. 
17. Cheng, Y. Q.; Ma, E.; Sheng, H. W. Atomic Level Structure in Multicomponent Bulk Metallic Glass. Phys. Rev. Lett. 2009, 102 (24), 245501.

18. Lu, Z.; Jiao, W.; Wang, W. H.; Bai, H. Y. Flow Unit Perspective on Room Temperature Homogeneous Plastic Deformation in Metallic Glasses. Phys. Rev. Lett. 2014, 113 (4), 045501.

19. Wang, Z.; Sun, B. A.; Bai, H. Y.; Wang, W. H. Evolution of hidden localized flow during glass-toliquid transition in metallic glass. Nat. Commun. 2014, 5, 5823.

20. Wang, Z.; Wen, P.; Huo, L. S.; Bai, H. Y.; Wang, W. H. Signature of viscous flow units in apparent elastic regime of metallic glasses. Appl. Phys. Lett. 2012, 101 (12), 121906.

21. Widmer-Cooper, A.; Perry, H.; Harrowell, P.; Reichman, D. R. Irreversible reorganization in a supercooled liquid originates from localized soft modes. Nat. Phys. 2008, 4 (9), 711-715.

22. Manning, M. L.; Liu, A. J. Vibrational Modes Identify Soft Spots in a Sheared Disordered Packing. Phys. Rev. Lett. 2011, 107 (10), 108302.

23. Wang, L.; Ninarello, A.; Guan, P.; Berthier, L.; Szamel, G.; Flenner, E. Low-frequency vibrational modes of stable glasses. Nat. Commun. 2019, 10 (1), 26.

24. Egami, T. Atomic level stresses. Prog. Mater. Sci. 2011, 56 (6), 637-653.

25. Egami, T.; Maeda, K.; Vitek, V. Structural defects in amorphous solids A computer simulation study. Philos. Mag. A 1980, 41 (6), 883-901.

26. Egami, T.; Srolovitz, D. Local structural fluctuations in amorphous and liquid metals: a simple theory of the glass transition. J. Phys. F: Met. Phys. 1982, 12 (10), 2141.

27. Patinet, S.; Vandembroucq, D.; Falk, M. L. Connecting Local Yield Stresses with Plastic Activity in Amorphous Solids. Phys. Rev. Lett. 2016, 117 (4), 045501.

28. Ding, J.; Patinet, S.; Falk, M. L.; Cheng, Y.; Ma, E. Soft spots and their structural signature in a metallic glass. Proc. Natl. Acad. Sci. 2014, 111 (39), 14052-14056. 
29. Xu, B.; Falk, M. L.; Li, J. F.; Kong, L. T. Predicting Shear Transformation Events in Metallic Glasses. Phys. Rev. Lett. 2018, 120 (12), 125503.

30. Ding, J.; Cheng, Y.-Q.; Sheng, H.; Asta, M.; Ritchie, R. O.; Ma, E. Universal structural parameter to quantitatively predict metallic glass properties. Nat. Commun. 2016, 7, 13733.

31. Makarov, A. S.; Afonin, G. V.; Mitrofanov, Y. P.; Konchakov, R. A.; Kobelev, N. P.; Qiao, J. C.; Khonik, V. A. Evolution of the activation energy spectrum and defect concentration upon structural relaxation of a metallic glass determined using calorimetry and shear modulus data. J. Alloys Compd. 2018, 745, 378-384.

32. Fan, Y.; Iwashita, T.; Egami, T. Energy landscape-driven non-equilibrium evolution of inherent structure in disordered material. Nat. Commun. 2017, 8, 15417.

33. Shi, Y.; Falk, M. L. Strain Localization and Percolation of Stable Structure in Amorphous Solids. Phys. Rev. Lett. 2005, 95 (9), 095502.

34. Cheng, Y. Q.; Ma, E. Atomic-level structure and structure-property relationship in metallic glasses. Prog. Mater. Sci. 2011, 56 (4), 379-473.

35. Wang, B.; Shang, B. S.; Gao, X. Q.; Wang, W. H.; Bai, H. Y.; Pan, M. X.; Guan, P. F. Understanding Atomic-Scale Features of Low Temperature-Relaxation Dynamics in Metallic Glasses. J. Phys. Chem. Lett. 2016, 7 (23), 4945-4950.

36. Hu, Y.-C.; Li, Y.-W.; Yang, Y.; Guan, P.-F.; Bai, H.-Y.; Wang, W.-H. Configuration correlation governs slow dynamics of supercooled metallic liquids. Proc. Natl. Acad. Sci. 2018, 115 (25), 6375. 37. Liu, C.; Guan, P.; Fan, Y. Correlating defects density in metallic glasses with the distribution of inherent structures in potential energy landscape. Acta Mater. 2018, 161, 295-301.

38. Ding, J.; Asta, M.; Ritchie, R. O. Anomalous structure-property relationships in metallic glasses through pressure-mediated glass formation. Phys. Rev. B 2016, 93 (14), 140204. 
39. Slipenyuk, A.; Eckert, J. Correlation between enthalpy change and free volume reduction during structural relaxation of $\mathrm{Zr}_{55} \mathrm{Cu}_{30} \mathrm{Al}_{10} \mathrm{Ni}_{5}$ metallic glass. Scripta Mater. 2004, 50 (1), 39-44.

40. Tanaka, H.; Kawasaki, T.; Shintani, H.; Watanabe, K. Critical-like behaviour of glass-forming liquids. Nat. Mater. 2010, 9 (4), 324-331.

41. Kawasaki, T.; Araki, T.; Tanaka, H. Correlation between Dynamic Heterogeneity and MediumRange Order in Two-Dimensional Glass-Forming Liquids. Phys. Rev. Lett. 2007, 99 (21), 215701.

42. Rieser, J. M.; Goodrich, C. P.; Liu, A. J.; Durian, D. J. Divergence of Voronoi Cell Anisotropy Vector: A Threshold-Free Characterization of Local Structure in Amorphous Materials. Phys. Rev. Lett. 2016, $116(8), 088001$.

43. Yang, J.; Wang, Y.-J.; Ma, E.; Zaccone, A.; Dai, L. H.; Jiang, M. Q. Structural Parameter of Orientational Order to Predict the Boson Vibrational Anomaly in Glasses. Phys. Rev. Lett. 2019, 122 (1), 015501.

44. Slotterback, S.; Toiya, M.; Goff, L.; Douglas, J. F.; Losert, W. Correlation between particle motion and Voronoi-cell-shape fluctuations during the compaction of granular matter. Phys. Rev. Lett. 2008, 101 (25), 258001.

45. Lacks, D. J.; Osborne, M. J. Energy Landscape Picture of Overaging and Rejuvenation in a Sheared Glass. Phys. Rev. Lett. 2004, 93 (25), 255501.

46. Kob, W.; Sciortino, F.; Tartaglia, P. Aging as dynamics in configuration space. Europhys. Lett. 2000, 49 (5), 590-596.

47. Tong, H.; Xu, N. Order parameter for structural heterogeneity in disordered solids. Phys. Rev. E 2014, 90 (1), 010401.

48. Liu, C.; Yan, X.; Sharma, P.; Fan, Y. Unraveling the non-monotonic ageing of metallic glasses in the metastability-temperature space. Comput. Mater. Sci. 2020, 172, 109347. 
49. Plimpton, S. Fast Parallel Algorithms for Short-Range Molecular Dynamics. J. Comp. Phys. 1995, 117, 1-19.

50. Cancès, E.; Legoll, F.; Marinica, M. C.; Minoukadeh, K.; Willaime, F. Some improvements of the activation-relaxation technique method for finding transition pathways on potential energy surfaces. J. Chem. Phys. 2009, 130 (11), 114711.

51. Barkema, G. T.; Mousseau, N. Event-Based Relaxation of Continuous Disordered Systems. Phys. Rev. Lett. 1996, 77 (21), 4358-4361. 

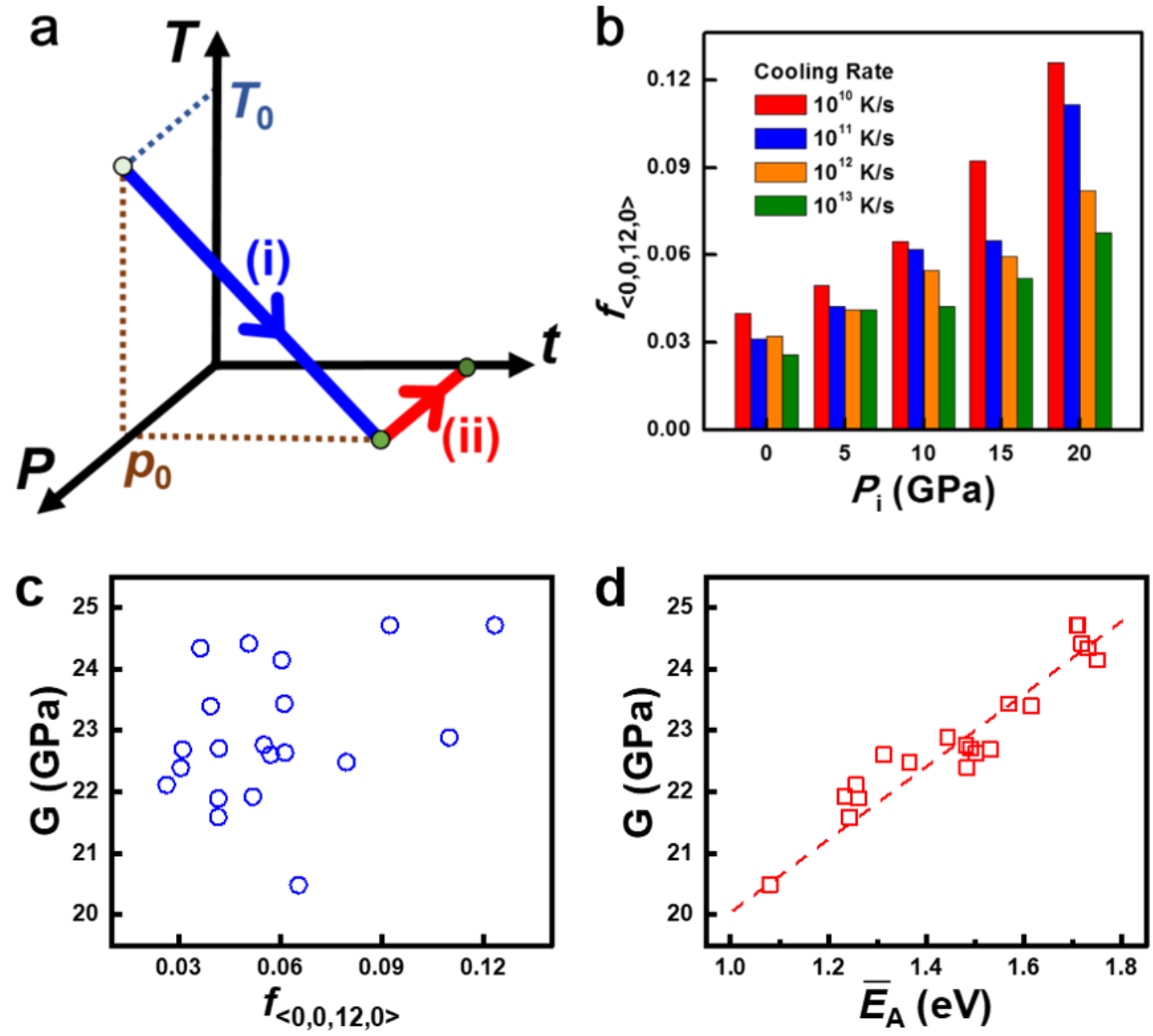

Figure 1 (a) Schematic diagram of the two-steps preparation process for each MG sample: (i) From $\mathrm{T}_{0}$ quenched to $1 \mathrm{~K}$ with different external pressures $\mathrm{P}_{\mathrm{i}}$ and cooling rates (ii) Released the pressures to 0 Gpa and kept the temperature at $1 \mathrm{~K}$ and relaxed the samples for 10ns. (b) The fraction of icosahedron cluster in investigated MG samples. The correlation between the shear modulus $\mathrm{G}$ and the $f<0,0,12,0>$ (c) and the the arithmetic mean of $E_{\mathrm{A}}$ spectra, $\bar{E}_{\mathrm{A}}(\mathrm{d})$. 

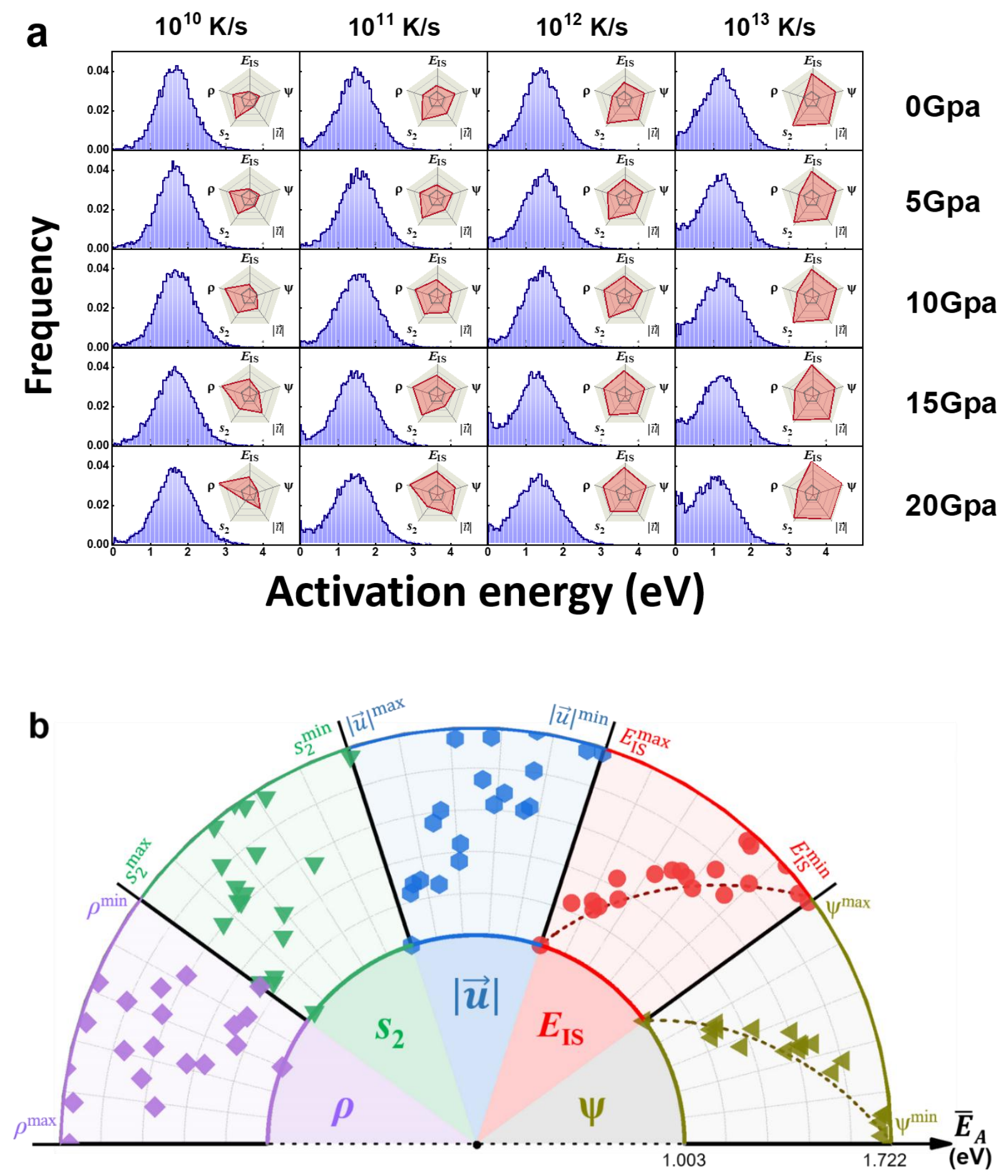

Figure 2 (a) The spectrum of $\mathrm{E}_{\mathrm{A}}$ for systems of various $\mathrm{P}_{i}$ and cooling rates. Each panel's inset with the radar plot shows the sample's $E_{\mathrm{IS}}, \Psi,|\vec{u}|, s_{2}$ and $\rho$, which scaled with the maximum value (raw data are shown in Fig.S2). (b) The average value of each parameter vs $\bar{E}_{\mathrm{A}}$ in polar coordinates. 

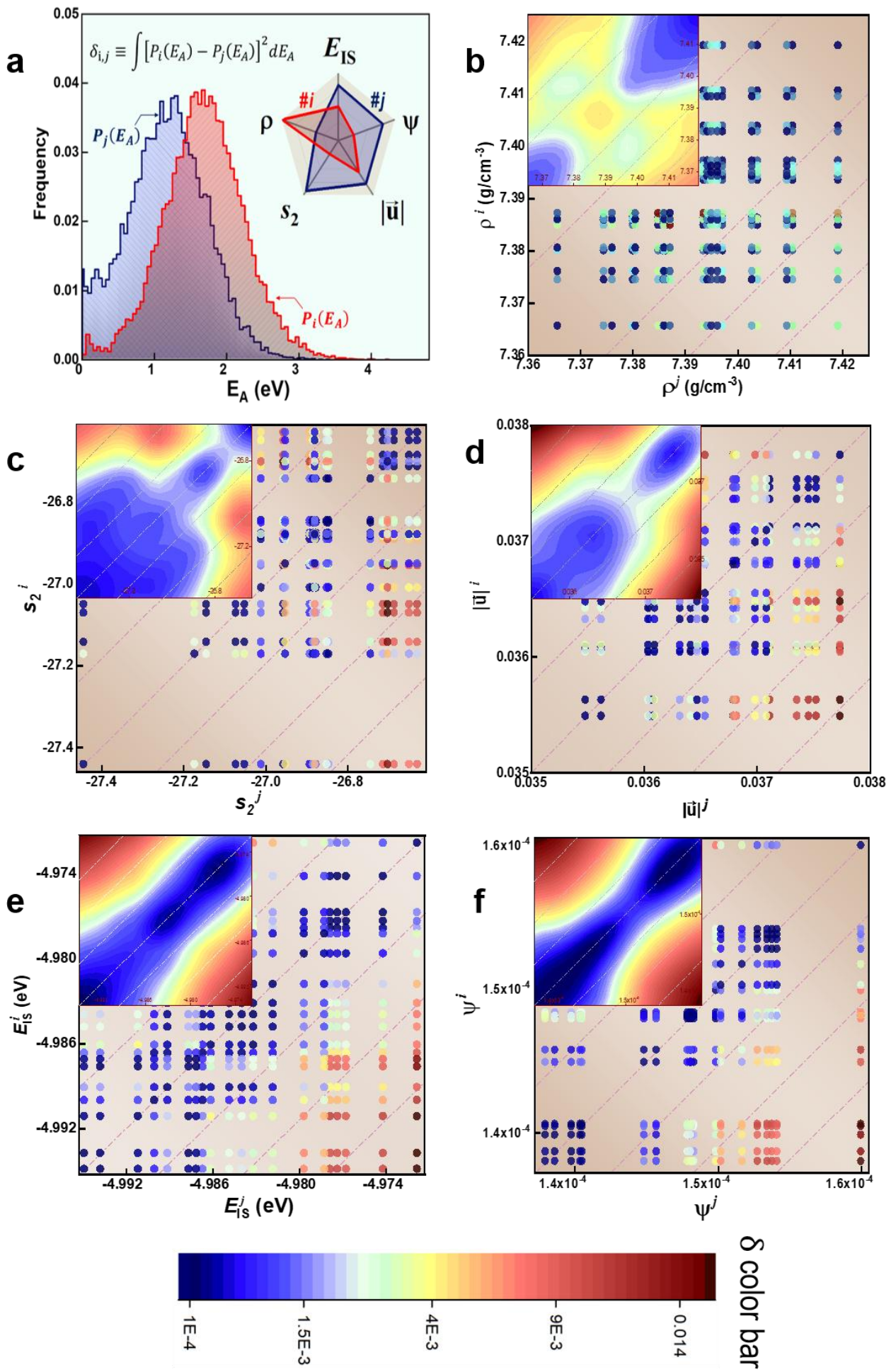
Figure 3 (a) The $E_{A}$ spectrum for the $i$ and $j$ systems. The difference of the $E_{A}$ spectra between $i$ and $j$ systems is defined as $\delta_{i, j}$. (b-f) Respective variations of $\delta_{i, j}$ dependence on $\rho, s_{2},|\vec{u}|, E_{\mathrm{IS}}$, and $\Psi$. The inset maps are contour maps of $\delta_{i, j}$.
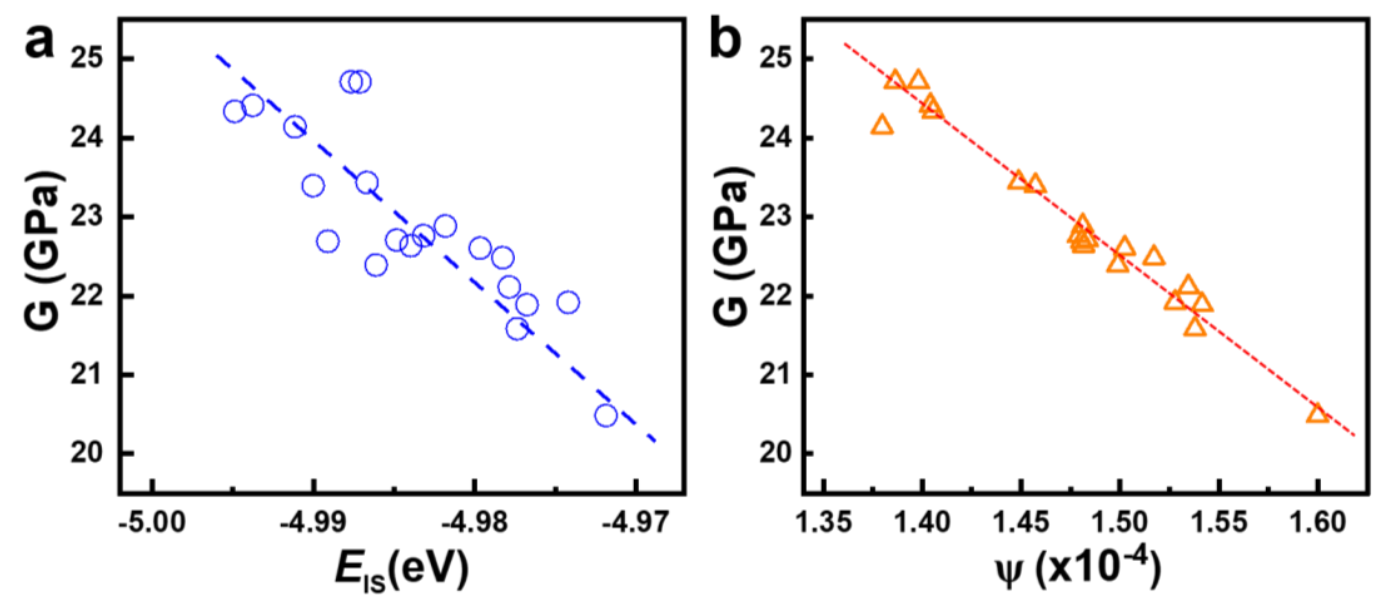

Figure 4 (a) The correlation between the shear modulus $G$ and the $E_{\mathrm{IS}}$ (a) and $\Psi(\mathrm{d})$. 


\title{
Supporting Information for
}

\section{Soft mode parameter as an indicator for the activation energy spectra in metallic glass}

\author{
Shan Zhang ${ }^{a}$, Chaoyi Liu $^{b}$, Yong Yang ${ }^{c}$, Yue Fan ${ }^{b}$, Pengfei Guan ${ }^{a, *}$ \\ ${ }^{a}$ Beijing Computational Science Research Center, Beijing, China \\ ${ }^{b}$ Department of Mechanical Engineering, University of Michigan, Ann Arbor, USA \\ ${ }^{c}$ Centre for Advanced Structural Materials, Department of Mechanical and Biomedical \\ Engineering, City University of Hong Kong, Hong Kong, China
}

\section{$1 \quad E_{A}$ spectrum and the arithmetic mean $\bar{E}_{\mathrm{A}}$}

$\mathbf{E}_{\mathbf{A}}$ spectrum. To obtain the $\mathbf{E}_{\mathbf{A}}$ spectrum of a given sample, we applied 10 activationrelaxation technique (ART) searches for each group of atoms. Thus, we can got the raw data by 40,000 ART searches for a given sample with 4000 atoms (center atom of a group). However, raw data contain failed and redundant searches. To acquire the accurate $\mathbf{E}_{\mathbf{A}}$ spectrum, we employed two steps based on four parameters to screen the failed and redundant searches.

Four parameters:

the energy difference between the saddle and initial state, $E_{\mathrm{sad}-\mathrm{ini}} \equiv E_{\mathrm{sad}}-E_{\mathrm{ini}}$ the energy difference between the final and saddle state, $E_{\text {fin-sad }} \equiv E_{\text {fin }}-E_{\text {sad }}$ the energy difference between the final and initial state, $E_{\mathrm{fin}-\mathrm{ini}} \equiv E_{\mathrm{fin}}-E_{\mathrm{ini}}$ the distance between the final state and initial state, $\Delta \equiv\left[\sum_{\mathrm{i}=1}^{\# \text { atoms }}\left(\overrightarrow{\mathrm{r}}_{\mathrm{fin}}^{\mathrm{i}}-\overrightarrow{\mathrm{r}}_{\text {ini }}^{\mathrm{i}}\right)^{2}\right]^{1 / 2}$

Two steps:

For each ART search,

1) 1) if $E_{\text {sad-ini }}<0$ or $E_{\text {fin-sad }}>0$, this event is a failed one and we will remove it. And then, if its $E_{\text {fin-ini }}<0.02(\mathrm{eV})$ and $\Delta<1(\AA)$, we will remove it(because in this event, the final state is identical to the initial state). After these steps, we have removed the failed and the final state the same as the initial searches. 
2) And then we go into the process of removing the redundant searches. For any pair of \#m and $\# n$ in the search that after steps 1$)$, if the $\left|\Delta^{\mathrm{m}}-\Delta^{\mathrm{n}}\right|<0.1(\AA)$, and $\left|E_{\text {sad-ini }}^{\mathrm{m}}-E_{\text {sad-ini }}^{\mathrm{n}}\right|$ $<0.01(\mathrm{eV})$, and $\left|E_{\text {fin-ini }}^{\mathrm{m}}-E_{\text {fin-ini }}^{\mathrm{n}}\right|<0.005(\mathrm{eV})$, we define them redundant and remove the redundant ones.

After these two removing steps, we have $\sim 25,000$ effective searches (in different samples, the number will be different ) with activation energy $E_{\mathrm{A}}$. Finally, we can obtain the distribution of $E_{\mathrm{A}}$, as the $\mathbf{E}_{\mathrm{A}}$ spectrum, for each sample.

The arithmetic mean $\overline{\boldsymbol{E}}_{\mathrm{A}}$. The $\bar{E}_{\mathrm{A}}$ is the arithmetic mean of all effective searches' activation energy $E_{\mathrm{A}}$ in each sample.

$$
\bar{E}_{\mathrm{A}}=\frac{1}{N_{\text {event }}} \sum_{i=1}^{N_{\text {event }}} E_{A}(i)
$$

where $N_{\text {event }}$ is the total number of valid events $(\sim 25000)$.

\section{The calculated total radial distribution functions (RDFs)}

For more information about the effects of different pressures and cooling rates on static structures, the total radial distribution functions RDFs of 20 samples prepared at four different cooling rates $\left(10^{10}\right.$ to $\left.10^{13} \mathrm{~K} / \mathrm{s}\right)$ and five different pressures $(0$ to $20 \mathrm{Gpa})$ are presented in Fig.S1 By comparing samples with varying cooling rates, we found that the changes in RDFs are not significant with the cooling rates. And the effects of stress are even more pronounced, although the external pressure has been released. At the same cooling rate, as the pressure increases, the shoulders of the first peak become gradually smooth and the splitting of the second peak becomes more pronounced. This shift shows that although the pressure is released, the pressure effect still retains some local geometric structure changes. The cooling rate has some impact on peak density. Combined with the above two factors, we can obtain 20 samples with very different local geometric structures. 

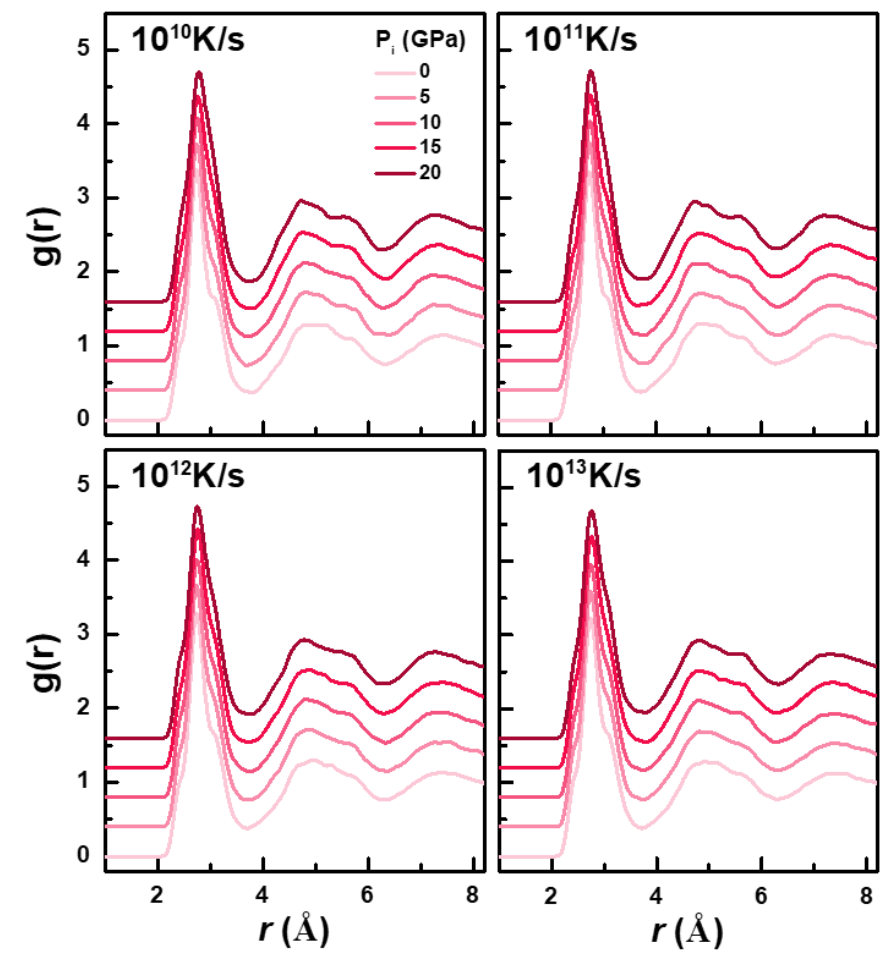

Fig.S1 The calculated radial distribution functions $g(r)$ of the samples at $10^{10} \sim 10^{13} \mathrm{~K} / \mathrm{s}$ cooling rates with various pressures (0 20Gpa).

\section{Local physical parameters at the atomic level}

Inherent structure energy $\boldsymbol{E}_{\mathrm{IS}}:$ Inherent structure energy $E_{\mathrm{IS}}$ is defined as the potential energy of the local minimum configuration of the potential energy landscape ${ }^{1}$. When the samples were prepared, we use the conjugate gradient (CG) algorithm method to get the minimized configuration and the intrinsic structure energy (per atom).

Local structural entropy $s_{2}$ : From the pair correlation function $g(r)$, we use the defined local structural entropy $s_{2}$ :

$$
s_{2, i}=-1 / 2 \sum_{v} \rho_{v} \int d \vec{r}\left\{g_{i}^{\mu \nu}(\vec{r}) \ln g_{i}^{\mu \nu}(\vec{r})-\left[g_{i}^{\mu \nu}(\vec{r})-1\right]\right\}
$$


Where $\rho_{\nu}$ is the number density of $v$ particle, $\nu, \mu$ is the particle types. Many works indicated the value of $s_{2, i}$ is correlated well with the slow dynamics ${ }^{2}$ and the heterogeneous dynamics ${ }^{3-4}$ of MGs. The average value $s_{2}$ of the sample:

$S_{2}=\left\langle\sum_{i=1}^{N} S_{2, i}\right\rangle$

where $\mathrm{N}$ is the number of atoms in the system.

Soft mode parameter $\Psi$ : The structure order parameter $\Psi$ is the participation ratio of low-frequency mode, correlating with the low-temperature dynamics and local surroundings Using the classical approximation and equipartition of energy to all vibration modes, the mean square vibrational amplitude of particle $i$ is defined by

$$
\Psi_{i}=\sum_{j=1}^{d N-d} \frac{1}{\omega_{j}^{2}}\left|\vec{e}_{j, i}\right|^{2}
$$

Where $\vec{e}_{j, i}$ is the polarization vector of particle $i$ of mode $j$ by the vibrational frequency $\omega$. Except for the $d$ zero-frequency modes, these are $d N-d$ modes in the system with $\mathrm{N}$ atoms. The low-frequency modes dominate the value of $\Psi_{i}$. It has a high correlation with Debye-waller factor and reflects the fast local dynamic properties of $\mathrm{MGs}^{5}$. Particles with large $\Psi_{i}$ have more opportunities for vibration and rearrangement. Given the overall, we used the average $\Psi$ :

$\Psi=\left\langle\sum_{i=1}^{N} \Psi_{\mathrm{i}}\right\rangle$

where $N$ is the number of atoms in the system.

Local configurational anisotropy $\left|\overrightarrow{\boldsymbol{u}_{\boldsymbol{\imath}}}\right|$ : The local configuration anisotropy is defined as the displacement from particle center to the corresponding Voronoi cell centroid. We usually use the Voronoi cell structures to define the local neighborhood of atoms in MGs. In this work, we set the modulus of the vector $\left|\overrightarrow{u_{\imath}}\right|^{6}$ is the displacement from the particle $i$ 's center to the average position of its Voronoi cell vertices. And it corresponds to the rearranging region space trend ${ }^{6-8}$. 


$$
|\vec{u}|=\left\langle\sum_{i=1}^{N}\left|\overrightarrow{u_{\imath}}\right|\right\rangle
$$

where the sample has $\mathrm{N}$ atoms.

4 The raw data of Figure $2 b$.

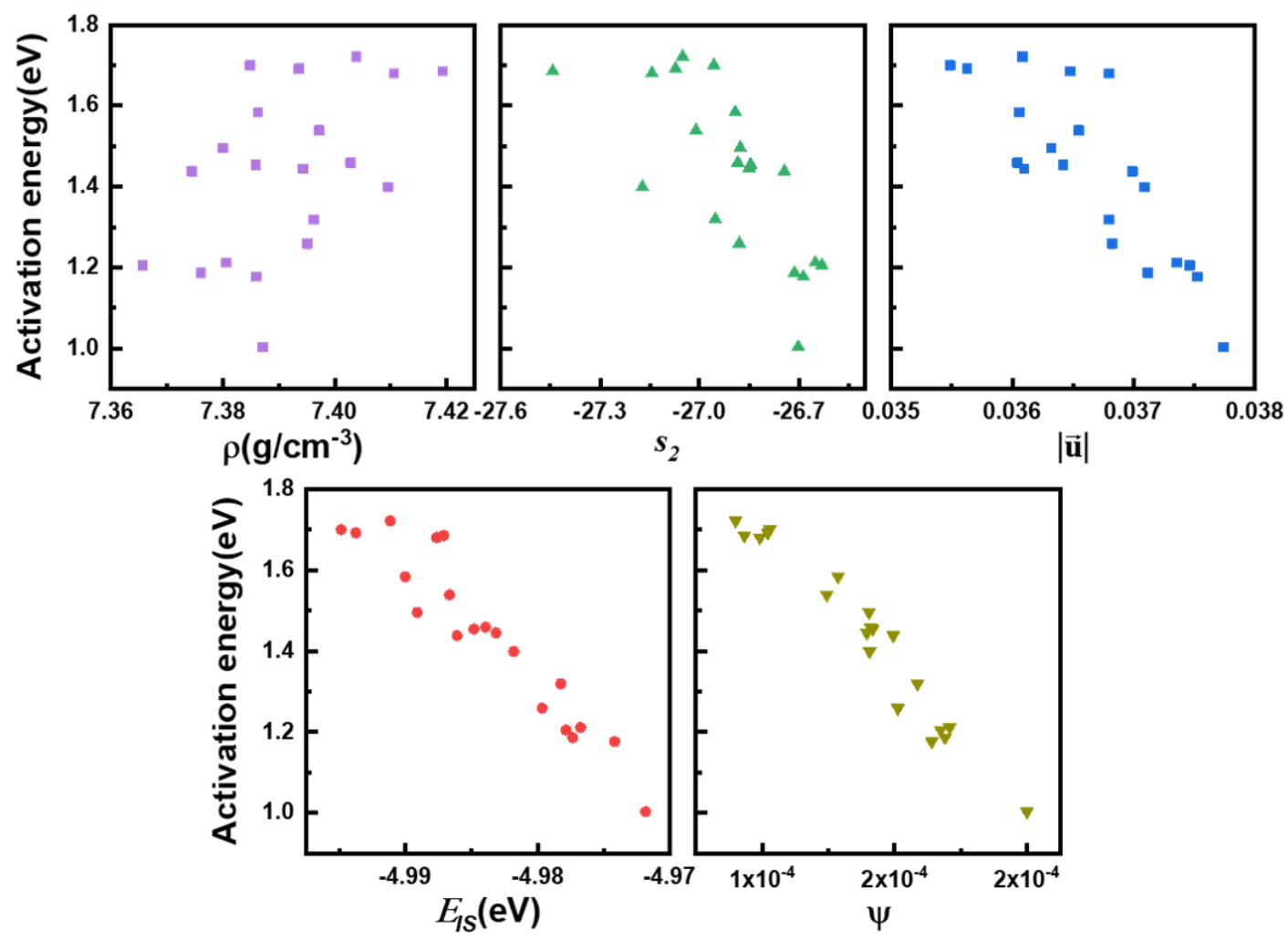

Fig.S2 The samples' average activation energy $\bar{E}_{\mathrm{A}}$ dependence on $\rho, E_{\mathrm{IS}}, s_{2}, \Psi$, and $|\vec{u}|$

\section{Details about the data analysis of Fig. 3.}

As shown in Fig.3, in each panel there are $400(=20 * 20)$ data entries, which are colored according to its $\delta$ value marked by the color bars. Note that not all the 400 points are independent, because the symmetry (i.e. $\left.P_{i j}=P_{j i}\right)$ and zero diagonals (i.e. $P_{i i}=0$ ) have to be satisfied due to the definition of Eq. (1). For the convenience of analyses, in the inset plots those discrete data points are converted into quasi-continuum contour maps using the thinplate spline (TPS) interpolation algorithm ${ }^{9}$. If there does exist one structural indicator that is able to characterize the $P\left(E_{\mathrm{A}}\right)$ with a minimum blurriness, then the contour map should 
possess the following two features: At first, the largest $\delta$ values should be concentrated near the up-left and bottom-right corners, because those extreme off-diagonal regions are where the structural differences between samples $\# i$ and $\# j$ are maximized; Secondly, the transition from the blue regime to the red regime should be continuous and smooth, so that it would ease the future development of an analytical expression of $P\left(E_{\mathrm{A}}\right)$. Following such spirit, Fig.

3e and Fig. 3f stand out among their peers, suggesting that $E_{\mathrm{IS}}$ and $\Psi$ are still the two best candidates correlating with $P\left(E_{\mathrm{A}}\right)$.

\section{References}

1. Sciortino, F.; Kob, W.; Tartaglia, P. Inherent Structure Entropy of Supercooled Liquids. Phys. Rev. Lett. 1999, 83.

2. Kawasaki, T.; Araki, T.; Tanaka, H. Correlation between Dynamic Heterogeneity and MediumRange Order in Two-Dimensional Glass-Forming Liquids. Phys. Rev. Lett. 2007, 99 (21), 215701.

3. Zheng, Z.; Ni, R.; Wang, F.; Dijkstra, M.; Wang, Y.; Han, Y. Structural signatures of dynamic heterogeneities in monolayers of colloidal ellipsoids. Nat. Commun. 2014, 5, 3829.

4. Zong, Y.; Chen, K.; Mason, T. G.; Zhao, K. Vibrational Modes and Dynamic Heterogeneity in a Near-Equilibrium 2D Glass of Colloidal Kites. Phys. Rev. Lett. 2018, 121 (22), 228003.

5. Yang, X.; Tong, H.; Wang, W.-H.; Chen, K. Emergence and percolation of rigid domains during the colloidal glass transition. Phys. Rev. E 2019, 99 (6), 062610.

6. Slotterback, S.; Toiya, M.; Goff, L.; Douglas, J. F.; Losert, W. Correlation between particle motion and Voronoi-cell-shape fluctuations during the compaction of granular matter. Phys. Rev. Lett. 2008, 101 (25), 258001.

7. Rieser, J. M.; Goodrich, C. P.; Liu, A. J.; Durian, D. J. Divergence of Voronoi Cell Anisotropy Vector: A Threshold-Free Characterization of Local Structure in Amorphous Materials. Phys. Rev. Lett. 2016, $116(8), 088001$.

8. Yang, J.; Wang, Y.-J.; Ma, E.; Zaccone, A.; Dai, L. H.; Jiang, M. Q. Structural Parameter of Orientational Order to Predict the Boson Vibrational Anomaly in Glasses. Phys. Rev. Lett. 2019, 122 (1), 015501.

9. Donato, G.; Belongie, S. In Approximate Thin Plate Spline Mappings, Computer Vision - ECCV 2002, Berlin, Heidelberg, 2002//; Heyden, A.; Sparr, G.; Nielsen, M.; Johansen, P., Eds. Springer, Berlin, Heidelberg: Berlin, Heidelberg, 2002; pp 21-31. 